\title{
HMGA2 expression in white adipose tissue linking cellular senescence with diabetes
}

\author{
Dominique Nadine Markowski • Helge Wilhelm Thies • \\ Andrea Gottlieb · Heiner Wenk - Manfred Wischnewsky • \\ Jörn Bullerdiek
}

Received: 30 May 2013/ Accepted: 12 July 2013/Published online: 24 July 2013

(C) Springer-Verlag Berlin Heidelberg 2013

\begin{abstract}
There is a clear link between overweight, gain of white adipose tissue, and diabetes type 2 (T2D). The molecular mechanism of the gain of adipose tissue is linked with the expression of high mobility group protein AT-hook 2 (HMGA2), and recent studies revealed an association with a SNP near $H M G A 2$. In this study, we investigated the gene expression of $H M G A 2, p 14^{A r f}, C D K N 1 A$, and $B A X$ in human abdominal subcutaneous white adipose tissue from 157 patients. We found a significant higher HMGA2 expression in obese individuals than in non-obese patients. Furthermore, the HMGA2 expression in white adipose tissue in patient with type 2 diabetes was significantly higher than in nondiabetic patients. There is an association between the DNA-binding nonhistone protein HMGA2 and
\end{abstract}

Dominique Nadine Markowski and Helge Wilhelm Thies have contributed equally.

Electronic supplementary material The online version of this article (doi:10.1007/s12263-013-0354-6) contains supplementary material, which is available to authorized users.

D. N. Markowski · H. W. Thies $(\bowtie) \cdot$ A. Gottlieb ·

J. Bullerdiek

Center of Human Genetics, University of Bremen,

Leobener Strasse ZHG, 28359 Bremen, Germany

e-mail: hthies@uni-bremen.de

J. Bullerdiek

e-mail: bullerd@uni-bremen.de

H. Wenk

Department of General and Vascular Surgery,

Clinical Center Bremen-Nord, Hammersbecker Str. 228,

28277 Bremen, Germany

M. Wischnewsky

Department of Mathematics and Computer Science, University

of Bremen, Universitätsallee, 28359 Bremen, Germany the risk of developing T2D that remains mechanistically unexplained so far. Likewise, $14^{\text {Arf }}$, an inducer of cellular senescence, has been associated with the occurrence of T2D. The data of the present study provide evidence that both proteins act within the same network to drive proliferation of adipose tissue stem and precursor cells, senescence, and increased risk of T2D, respectively.

Keywords HMGA2 - Subcutaneous abdominal white adipose tissue $\cdot$ Type 2 diabetes $\cdot$ ADSCs $\cdot$ p1 $4^{\text {Arf }}$

\section{Introduction}

Though there is little doubt about the association between overweight, gain of white adipose tissue (WAT), and diabetes type 2 (T2D), the mechanisms by which an excess of adipose tissue contributes to the onset of the metabolic syndrome are as yet far away from being fully understood (Ahima 2009). However, chronic inflammation contributes to a tissue environment that triggers an accelerated aging process and insulin resistance (Ahima 2009; Minamino et al. 2009). Some recent evidence suggests that an accumulation of immature senescent cells irreversibly unable to re-enter the cell cycle can lead to inflammatory processes (Tchkonia et al. 2010). A protein that is apparently linked to the process of proliferation and differentiation of adipose tissue stem and progenitor cells is high mobility group protein AT-hook 2 (HMGA2). Its gene (HMGA2) maps to a segment of $12 \mathrm{q} 14 \sim 15$ that is rearranged in a variety of tumors mostly of mesenchymal origin, including lipomas (Ashar et al. 1995; Schoenmakers et al. 1995a), pulmonary chondroid hamartomas, uterine leiomyomas, and fibroadenomas of the breast (Kazmierczak et al. 1999; Hennig et al. 1996; Schoenmakers et al. 1995b; Staats et al. 1996). 
These rearrangements result in the formation of fusion genes, e.g., those with LPP (Rogalla et al. 2000) or merely in a strong transcriptional up-regulation of HMGA2 (Bartuma et al. 2009; Tallini et al. 1997) likely causing tumorigenesis. As to normal tissues, there are strong experimental data linking HMGA2 with hyperplasia of WAT. Transgenic mice expressing truncated HMGA2 still retain the three AT-hook domains present with a giant phenotype and hyperplasia of WAT (Battista et al. 1999). Vice versa, HMGA2 knockout mice show a pygmy phenotype with hypoplasia of WAT (Zhou et al. 1995). Likewise, a lack of HMGA2 impairs lineage commitment of stem cells toward pre-adipocytes (Pasquali et al. 2004). Ayoubi et al. (1999) were able to show that in the murineimmortalized cell line NIH3T3, HMGA2 can be induced by several growth factors as well as by fetal calf serum. Moreover, even normal not immortalized cells from adult adipose tissue have been shown to be able to execute a HMGA2 response, i.e., appropriate stimuli lead to a short abundant expression of $H M G A 2$. In a recent study, an increased "HMGA2-response" was found to be associated with overweight in dogs (Thies et al. 2013). This challenges earlier findings that HMGA2 is a protein normally almost exclusively expressed during embryonic and fetal life. Apparently, many types of stem cells of adult mammals are able to restore the expression of $H M G A 2$ which in these cases might reflect self-renewal activity of stem and progenitor cells.

While the expression of $H M G A 2$ has been linked to a gain of adipose tissue and some recent studies revealed an association between variants of $H M G A 2$ and different risks to develop T2D (Ohshige et al. 2011; Voight et al. 2010; Dayeh et al. 2013), the exact mechanisms linking HMGA2 with T2D remain obscure. A possibly relevant pathway addressing HMGA2's role in WAT links the CDKN2A locus with the activation of $H M G A 2$ that, in adipose tissuederived stem cells (ADSCs), recently has been associated with the induction of one of the two genes of the senescence-associated CDKN2A locus, i.e., p14 $4^{\text {Arf }}$ (Markowski et al. 2011a), an upstream agonist of p53 driving apoptosis and senescence. Since the main players within that network are well-conserved among mammals, these latter effects can be considered being part of an ancient mechanism preventing activated mesenchymal stem cells from undergoing malignant transformation. On the other hand, an increased expression of $H M G A 2$ can be expected to result in an increase in the expression of $p 14^{A r f}, p 53$, and CDKN1A and subsequently an increase in senescent cells (Markowski et al. 2011a). Generally, an increase in senescent cells is believed to contribute to age-related diseases. For example, in mice, excessive caloric intake promoted senescence-associated changes like an increased $\beta$-galactosidase activity, an increased expression of p53 and of pro-inflammatory cytokines finally leading to insulin resistance (Minamino et al. 2009). As a mechanism, the accumulation of oxidative stress has been proposed. In addition, the increased proliferation of adipose tissue stem and progenitor cells may lead to their increased probability to enter senescence. Therefore, we were interested to investigate whether the link between the up-regulation of $H M G A 2$ and an increased expression of $p 14^{A r f}$ and its downstream effectors of senescence and apoptosis as revealed by in vitro studies can be confirmed on native human tissues.

\section{Materials and methods}

Tissue samples

Human subcutaneous abdominal WAT from 157 patients used in this study was taken during surgery and immediately frozen in liquid nitrogen. Prior to surgery, informed written consent was obtained from all patients. In addition, the study has been approved by the local ethics committee (Ethikkomission der Ärztekammer Bremen, no. 309). Clinical data were obtained by data-recording sheets.

\section{RNA isolation}

Total RNA was isolated using an RNeasy mini kit (Qiagen, Hilden Germany) in a QIACube (Qiagen) according to the manufacturer's instructions, and DNase I digestion was performed.

\section{cDNA-synthesis}

About $250 \mathrm{ng}$ of total RNA were reverse transcribed with $200 \mathrm{U}$ of M-MLV reverse transcriptase (Invitrogen, Darmstadt, Germany), RNase Out, and $150 \mathrm{ng}$ random hexamers according to the manufacturer's instructions. RNA was denatured at $65^{\circ} \mathrm{C}$ for $5 \mathrm{~min}$ and subsequently kept on ice for $1 \mathrm{~min}$. After adding the enzyme to the RNA primer mixes, samples were incubated for $10 \mathrm{~min}$ at $25{ }^{\circ} \mathrm{C}$ to allow annealing of the random hexamers. Reverse transcription was performed at $37{ }^{\circ} \mathrm{C}$ for $50 \mathrm{~min}$ followed by inactivation of the reverse transcriptase at $70{ }^{\circ} \mathrm{C}$ for $15 \mathrm{~min}$.

\section{Quantitative real-time PCR}

Relative quantification of transcription levels was carried out on the samples by real-time PCR analyses using the Applied Biosystems 7300 real-time PCR system (Applied Biosystems, Darmstadt, Germany). Commercially available gene expression assays (Applied Biosystems) were 
used for quantification of mRNA of $p 14^{A r f}$ (Hs00924091), CDKN1A (Hs99999142), BAX (Hs00180269), and HMGA2 (Hs00171569). HPRT served as endogenous control as described before (Markowski et al. 2010). All qRT-PCR experiments were done in triplicate.

\section{Statistical evaluation}

Nominally scaled variables were tabulated in contingency tables and tested for differences in frequency distribution. Variables for location and variance were calculated, whenever ordinally scaled and intervally scaled continuous variables were involved. For each group, we calculated the following: number of cases, mean, standard deviation, standard error of the mean, minimum, maximum, and $95 \%$ confidence interval (CI) for the mean, medians [interquartile range, IQR], Levene's test for homogeneity of variance, analysis-of-variance table, and robust tests of the equality of means (medians). Normal distribution was tested using the Kolmogorov-Smirnov goodness-of-fit test. In addition to the standard Kolmogorov-Smirnov test, we also used the Anderson-Darling test and the Cramer-Smirnov-Von-Mises test, a modification of the $\mathrm{K}-\mathrm{S}$ test, giving more weight to the tails of the distribution than does the $\mathrm{K}-\mathrm{S}$ test. In order to compare the distributions and medians of the various biomarkers across diabetes, we used the independent samples Kolmogorov-Smirnov test, the Kruskal-Wallis test, the Mann-Whitney $U$ test, and the median test. Correlations between the mRNA biomarkers were analyzed using Kendall's Tau-b correlations. Decision trees (Exhausted CHAID or CART) were calculated in order to create models that predict the value of one of the biomarkers as a target variable based on several other biomarkers or basic characteristics as input variables.

Beside decision trees to uncover interactions within the HMGA2-p14 ${ }^{\text {Arf }}$-p53 network, we used the data of the expression analyses as well as BMI and the diagnosis of T2D to create self-organizing maps (SOM) (Kohonen 2001). SOM may be considered a nonlinear generalization of principal components analysis (PCA). A SOM is a type of artificial neural network that is trained using unsupervised learning to produce a low-dimensional (typically two-dimensional), discretized representation of the input space of the training samples and called a map. The map learns the typical features of the subclasses in the learning set by means of a shortest Euclidean distance algorithm, after which self-adaptation of the neurons occurs. SOM has many advantages over the conventional feature extraction methods such as empirical orthogonal functions or PCA. SOM has been interpreted using semantic maps where similar samples are mapped close together and dissimilar ones apart.
Calculations were performed with NCSS (version 2007), SPSS (version 20), R (2.15.3), and Viscovery (version 5.1).

\section{Results}

Kendall's Tau-b correlation test revealing correlations between the expression of $H M G A 2, p 14^{A r f}, B A X$, and $C D K N 1 A$

Median age of patients was 65 years (range 19-97). $19.1 \%$ were older than 75 years. $32.5 \%$ had overweight (BMI 25.0-30.0), $20.4 \%$ had adipositas (BMI > 30.0), and $10.2 \%$ had been diagnosed as being diabetic patients (Table 1). HMGA2, p14 ${ }^{\text {Arf }}$, BAX, and CDKN1A mRNA expressions were detectable in all 157 samples. The relative levels of HMGA2 mRNA ranged between 0.11 and 2.66 with mean $0.72 \pm 0.48$ (Fig. 1). The distributions of p14 ${ }^{A r f}, H M G A 2$, and CDKN1A expressions were not normal (One-Sample-Kolmogorov-Smirnov test) (suppl. Fig. 1a-c, suppl. Tab. 1). Only $B A X$ was normally distributed with an asymptotic significance (2-tailed) of 0.634 . Akin to what had been observed in in vitro studies, there were weak correlations at the 0.05 level (2-tailed) (Kendall's Tau-b) between any pair of the four determined biomarkers (Table 2). As to HMGA2 mRNA, there was even a weak correlation at the 0.01 level (2-tailed) with that of p14Arf and CDKN1A.

\section{HMGA2 and obesity}

Obesity (BMI $>25 \mathrm{~kg} / \mathrm{m}^{2}$ ) was significantly associated with an increase in the expression of HMGA2 mRNA in our series of samples of human WAT. The Mann-Whitney $U$ test showed that the distribution of HMGA2 mRNA was significantly ( $p=0.044)$ different across the two categories of obese and non-obese patients. The median rank of HMGA2 mRNA for obese patients was 85.9 and 71.3 for non-obese patients. The mean of $H M G A 2$ expression was $0.81 \pm 0.55$ in the group of obese patients $(n=83$; $52.9 \%)$ and $0.62 \pm 0.38$ in the group of non-obese patients $(n=74 ; 47.1 \%)(p=0.016$; exhausted CHAID).

A significantly higher expression of HMGA2 mRNA in adipose tissue in patients with type 2 diabetes

$16(10.3 \%)$ patients had a previous diagnosis of T2D. Diabetes was found to be highly significantly $(p<0.001)$ associated with an increase in the expression of HMGA2 mRNA. The independent samples Mann-Whitney U test (as well as the independent samples Kruskal-Wallis test) showed that the distribution of HMGA2 mRNA was highly significantly $(p=0.001)$ different across the two 
Table 1 Characteristics of the human subcutaneous abdominal white adipose tissue donors

\begin{tabular}{ll}
\hline & $\begin{array}{l}n=157 \\
\text { Number }(\%)\end{array}$ \\
\hline Sex & \\
Female & $66(42.0)$ \\
Male & $91(58.0)$ \\
Age & \\
Median & 65.0 years \\
Min & 19 years \\
Max & 97 years \\
Age group & \\
$<65$ years & $83(52.9)$ \\
$65-75$ years & $44(28.0)$ \\
$>75$ years & $30(19.1)$ \\
BMI & \\
Underweight $(<18.5)$ & $1(0.6)$ \\
Normal weight $(18.5-25.0)$ & $73(46.5)$ \\
Overweight $(25.0-30.0)$ & $51(32.5)$ \\
Adipositas $(>30.0)$ & $32(20.4)$ \\
Diabetes & \\
Yes & $16(10.2)$ \\
Biomarker $($ mean \pm sd; min, max $)$ & \\
p14 ${ }^{\text {Arf }}$ & $1.34 \pm 1.06 ; 0.14,0.90$ \\
BAX & $0.79 \pm 0.16 ; 0.48,1.67$ \\
p21 & $1.38 \pm 0.59 ; 0.50,4.12$ \\
HMGA2 & $0.72 \pm 0.48 ; 0.11,2.66$ \\
\hline
\end{tabular}



Fig. 1 Histogram showing the distribution of 157 human abdominal subcutaneous adipose tissue samples and their relative $H M G A 2$ expression. The housekeeping gene HPRT served as reference for relative quantification. One-sample Kolmogorov-Smirnov test revealed that the test distribution is not normal (asymptotic significance $(2$-tailed $)=0.019)$
Table 2 Kendall's Tau-b statistic test revealed correlation between the expression of HMGA2, p14 ${ }^{\text {Arf }}$, p21, and Bax

\begin{tabular}{lllll}
\hline & $\mathrm{p} 14^{\text {Arf }}$ & BAX & p21 & HMGA2 \\
\hline$p 14^{\text {Arf }}$ & & & & \\
Correlation Coefficient & 1 & $0.333^{\mathrm{a}}$ & $0.188^{\mathrm{a}}$ & $0.180^{\mathrm{a}}$ \\
Sig. (2-tailed) & & $<0.001$ & 0.003 & 0.001 \\
$N$ & 157 & 157 & 157 & 157 \\
$B A X$ & & & & \\
Correlation Coefficient & $0.333^{\mathrm{a}}$ & 1 & $0.265^{\mathrm{a}}$ & $0.127^{\mathrm{b}}$ \\
Sig. (2-tailed) & $<0.001$ & & $<0.001$ & 0.048 \\
$N$ & 157 & 157 & 157 & 157 \\
$p 21$ & & & & \\
Correlation Coefficient & $0.188^{\mathrm{a}}$ & $0.265^{\mathrm{a}}$ & 1 & $0.272^{\mathrm{a}}$ \\
Sig. (2-tailed) & 0.003 & $<0.001$ & & $<0.001$ \\
$N$ & 157 & 157 & 157 & 157 \\
$H M G A 2$ & & & & \\
Correlation Coefficient & $0.180^{\mathrm{a}}$ & $0.127^{\mathrm{b}}$ & $0.272^{\mathrm{a}}$ & 1 \\
Sig. (2-tailed) & 0.001 & 0.048 & $<0.001$ & \\
$N$ & 157 & 157 & 157 & 157 \\
\hline
\end{tabular}

a Correlation is significant at the 0.01 level (2-tailed)

${ }^{\mathrm{b}}$ Correlation is significant at the 0.05 level (2-tailed)

categories of diabetic and nondiabetic patients. The median rank of HMGA2 mRNA for diabetic patients was 115.6 and 74.3 for nondiabetic patients. The mean of $H M G A 2$ expression was $1.24 \pm 0.68$ in the group of diabetic patients and $0.65 \pm 0.42$ the group of nondiabetic patients $(p<0.001$; exhausted CHAID) (Fig. 2).

In mesenchymal stem cells (MSC), as well as in uterine leiomyomas, the increase in HMGA2 was found to be accompanied by an increase in p14 Arf (Markowski et al. 2011a). Due to the fact that MSCs are part of WAT, we analyzed the distribution of $\mathrm{p} 14^{\text {Arf }}$ in WAT across 140 nondiabetics and 16 diabetics. The independent samples Mann-Whitney $U$ test showed that the distribution of

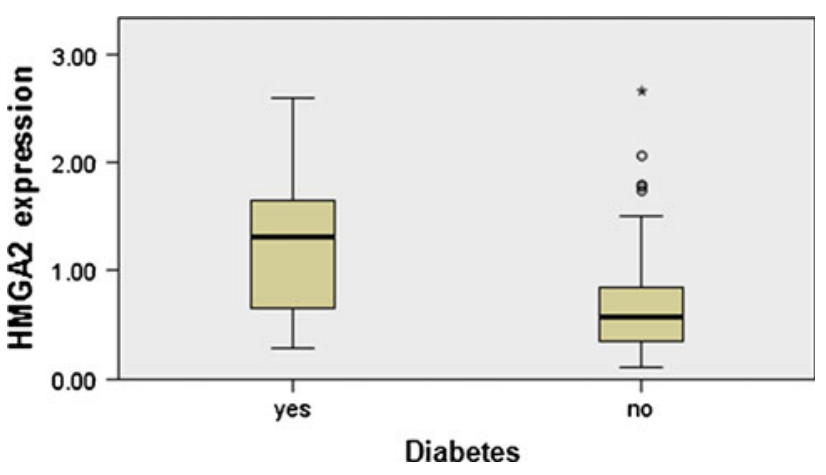

Fig. 2 Boxplot showing the relative HMGA2 expression in WAT from patients with T2D $(n=16)$ and nondiabetic patients $(n=140)$. Independent samples Kruskall-Wallis test revealed a highly significant $(p<0.001)$ elevated expression of HMGA2 in patients with T2D. The test statistics is adjusted for ties 


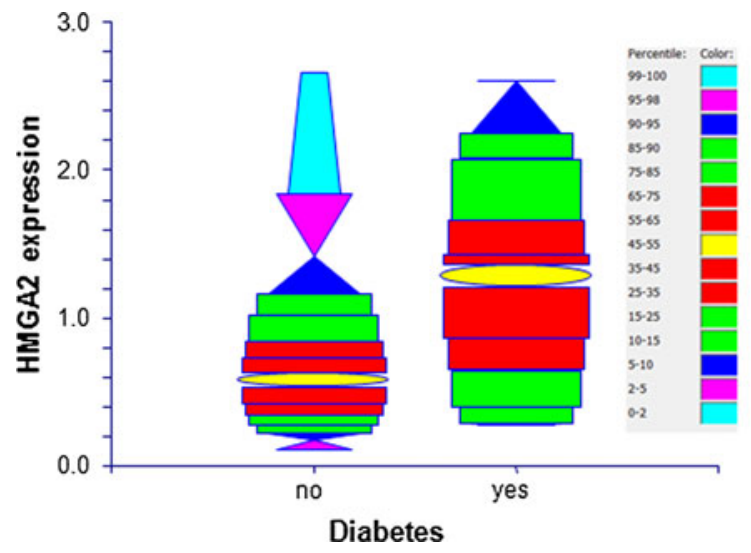

Fig. 3 Percentile plots of relative expression of $H M G A 2$ and $p 14^{A r f}$ in WAT from patients with T2D $(n=16)$ and nondiabetic patients $(n=140)$. In almost every percentile, the diabetic group showed a higher gene expression than in their corresponding percentile in the

$p 14^{A r f}$ expression is significantly $(p=0.020)$ different across the two categories of diabetic and nondiabetic patients. The mean rank of $\mathrm{p} 14^{\mathrm{Arf}} \mathrm{mRNA}$ for diabetic patients was 103.5 and 75.7 for nondiabetic patients. The mean of $\mathrm{p} 14^{\text {Arf }}$ mRNA expression was $1.97 \pm 1.94$ in the group of diabetic patients and $1.27 \pm 0.90$ in the group of nondiabetic patients ( $p=0.012$; exhausted CHAID).

The percentile plots from a set of 15 percentiles $(0-2$, $2-5,5-10,10-15,15-25,25-35,35-45,45-55,55-65$, $65-75,75-85,85-90,90-95,95-99,99-100)$ for HMGA2 and $\mathrm{p} 14^{\text {Arf }}$ mRNA, an estimate of the corresponding distribution functions, show that in almost every percentile, the diabetic group has a higher gene expression than in their corresponding percentile in the nondiabetic group (Fig. 3).

Data analyses by self-organizing maps suggest a T2D risk profile associated with high $H M G A 2$ expression in a subset of diabetic individuals

We used Kohonen's self-organizing maps to analyze the interactions of the biomarkers HMGA2, p14 ${ }^{\text {Arf }}, \mathrm{p} 21$, and BAX with the BMI- and diabetes status in a multivariate model. Figure 4a shows the corresponding segmentations of the patients into 3 groups. The goal of this segmentation was to separate the patients into such groups (segments) that the variance of the attribute values in a segment was as small as possible and in between the groups as large as possible. In segment S3 were most of the patients with diabetes, and a much smaller cluster of diabetic patients could be found in segment S1 (Fig. 4c). The map for HMGA2 (Fig. 4b) shows the increase in the expression of HMGA2 mRNA for diabetic patients in S3. The patients in segment S2 with an expression of HMGA2 mRNA $>1.6$ might be those patients with pre-diabetes. Figure 5



nondiabetic group. Set of 15 percentiles $(0-2,2-5,5-10,10-15$, $15-25,25-35,35-45,45-55,55-65,65-75,75-85,85-90,90-95$, 95-99, 99-100)

represents the deviation of the mean of the values, an attribute of a segment from the mean of the corresponding attribute of the entire data set. S3 was characterized by a significant increase in the expressions of the biomarkers in question. The segment S2 was characterized by a light increase, whereas $\mathrm{S} 1$ was characterized by a light decrease. The mean value of HMGA2 mRNA was 1.4 in S3, 0.9 in $\mathrm{S} 2$, and 0.5 in $\mathrm{S} 1$, whereas the mean value for $\mathrm{p} 14^{\mathrm{Arf}}$ was 2.2 in $\mathrm{S} 3,1.6$ in $\mathrm{S} 2$, and 0.9 in $\mathrm{S} 1$.

\section{Discussion}

A gain of WAT is a hallmark of obesity. Nevertheless, details as to how diet-induced hypertrophy and hyperplasia of WAT are linked with the biology of mature adipocytes as well as with their precursors and stem cells are not fully understood, yet. Among the genes that have been linked to a gain of WAT and to T2D by large association studies (Ohshige et al. 2011; Voight et al. 2010; Zeggini et al. 2007) is HMGA2 which has been linked to the development and differentiation of WAT as well. Recently, we had been able to identify an association between the expression of HMGA2 and canine obesity (Thies et al. 2013). Based on these finding, we have hypothesized that the temporal activation of $H M G A 2$ in adipose tissue stem cells is a prerequisite not only of normal turnover of adipose tissue but also of its diet-induced hyperplasia. Since in vitro, the activation of $H M G A 2$ in ADSC by growth factors leads to an increasing expression of $p 14^{A r f}$ and accordingly of p53 downstream targets as in particular CDKNIA and $B A X$ we had aimed the present study at the question if comparable findings can be obtained on human WAT in vivo as well. The results confirm the hypothesis that the presumed associations between HMGA2 and $\mathrm{p} 14^{\text {Arf }}$ and its 
a

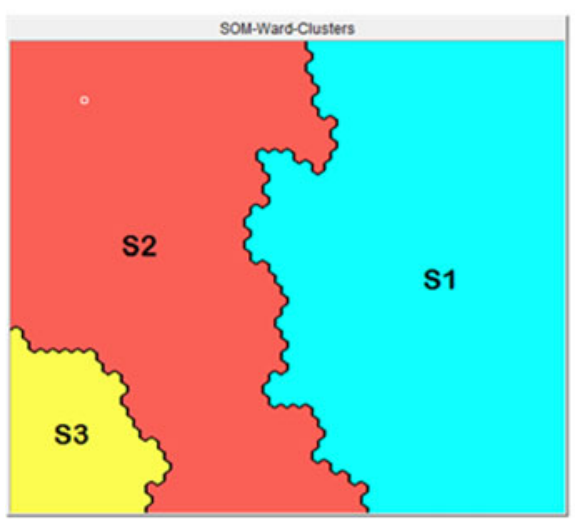

b

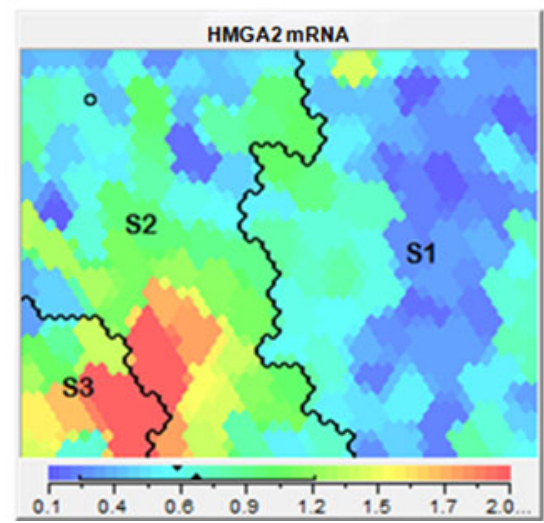

c



Fig. 4 Self-organizing maps suggests a T2D risk profile associated with high HMGA2 expression. a Distribution of subgroups (S1-S3) after creation of self-organized map. b Self-organized map of relative

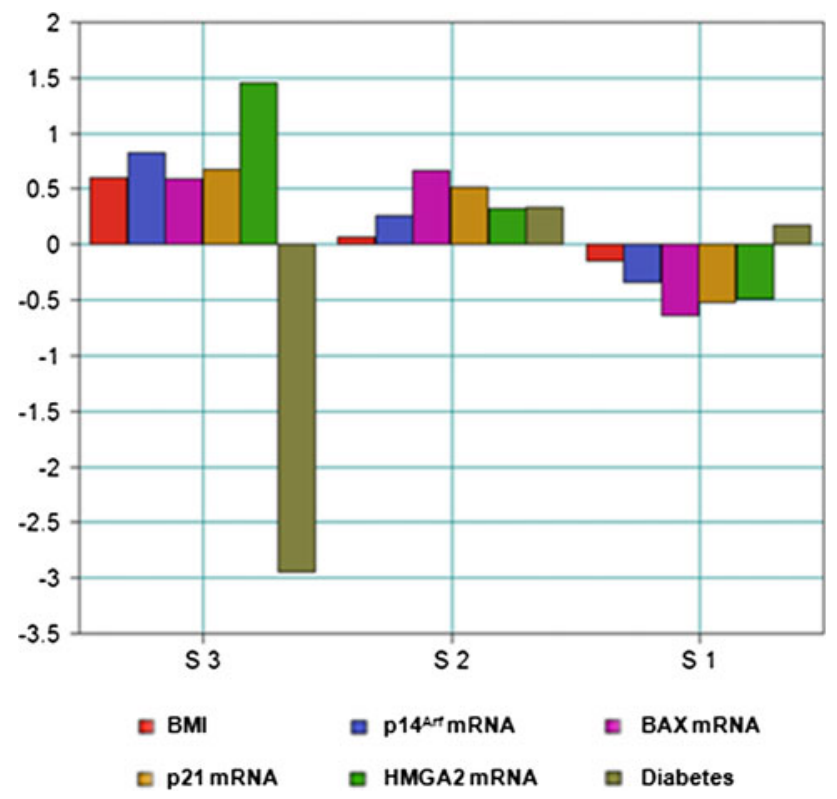

Fig. 5 Bar chart of the attributes in the three segments. The height of a bar in the bar chart is not the absolute mean of the values of an attribute of a segment, but the deviation of this mean from the mean of the entire data set. The unit is standard deviations of the entire data set. The diabetes status is inversely plotted, i.e., a negative value represents patients with T2D and a positive value nondiabetic patients

downstream targets were also detected in that large series of human WAT. Most likely, the increased level of HMGA2 does not result from a generally increased expression in mature adipocytes but from the activation in single stem and precursor cells. This makes it tempting to speculate that these latter cells are also prone to undergo cellular senescence. An accumulation of senescent cells has repeatedly been hypothesized to cause inflammation of adipose tissue finally contributing to insulin resistance (Ahima 2009; Minamino et al. 2009). From the results reported in the literature as well as from this study, there is
HMGA2 expression (low expression = blue; high expression $=$ red $)$. c Self-organized map of diabetic patient distribution (nondiabetic patient $=$ red; diabetic patient $=$ blue $)$

ample evidence that a gain of fat cells depends on the activation of $H M G A 2$. First, the constitutive activation of HMGA2, e.g., by chromosomal rearrangements causes lipomas, i.e., benign adipocytic tumors (Ashar et al. 1995; Schoenmakers et al. 1995a). Likewise, transgenic mice constitutively expressing $H M G A 2$ have been reported to develop subcutaneous lipomas and generally a drastic gain of adipose tissue (Battista et al. 1999). In contrast, constitutional knockout of HMGA2 is associated with a hypoplasia of WAT and a lack of an obese phenotype despite leptin deficiency (Zhou et al. 1995). Accordingly, we have proposed that the temporarily strictly scheduled activation of $H M G A 2$ distinguishes the processes in normal adult tissue from the monoclonal gain of adipose tissue underlying the development of lipomas (Thies et al. 2013). However, all these results point to a pivotal role of HMGA2 in the gain of adipocytes from their precursors and stem cells, respectively. On the other hand, activation of $H M G A 2$ seems to coincide with an increased expression of $p 14^{A r f}$ probably reflecting a mechanism protecting mesenchymal stem cells against malignant transformation (Markowski et al. 2011b). Subsequently, this increases the cell's probability to undergo cellular senescence. In turn, an accumulation of senescent cells is known to trigger inflammation known to be associated with insulin resistance, and more general with the metabolic syndrome. Of note, a high expression of HMGA2 in the present study was not always associated with T2D. Nonetheless, from their position on the map (cf. Fig. 4b), these individuals were close to the patients with known T2D (cf. Fig. 4c). Basically, two explanations fit with these findings. First, the island with high HMGA2 expression in a sector of $\mathrm{S} 2$ adjacent to the diabetes patients in S3 may in part represent T2D patients not yet detected. It is estimated that among the German population, roughly $50 \%$ of the T2D patients are not yet diagnosed (Rathmann et al. 2003). Moreover, 
this group of HMGA2 "high expressors" in S2 could also contain individuals with insulin resistance not yet displaying T2D. Thus, one might speculate whether an increased expression of HMGA2 mRNA in WAT can help to identify individuals at risk to become a diabetic patient. To some extent, the differences in HMGA2 expression may reflect genetic factors, but to a much larger extend, they can be expected to depend on the individual lifestyle with food intake and mobility being major determinants. Dynamics of $H M G A 2$ expression over time were not in the focus of the present study, and the sampling procedure accordingly did not address that point. Nevertheless, in a future study, repeated samples taken from individuals at different times should be analyzed in order to understand if and how fast changes of the lifestyle can influence HMGA2 expression in WAT.

From in vitro experiments, it has become evident that up- and downregulation of HMGA2 in ADSCs occurs depending on the level of growth factors (Markowski et al. 2011b). If it holds true that the differences of HMGA2 obsessed in WAT are due to changes in the stem and progenitor cell compartment, the kinetics may be similar to those observed in vitro with a relatively fast adjustment reacting to changes in food uptake, etc. Interestingly, in a recent paper, SNPs of HMGA2 and CDKN2A known to undergo epigenetic modifications have been associated with T2D (Dayeh et al. 2013) offering further evidence linking T2D with HMGA2-induced proliferation and cellular senescence, i.e., irreversible escape from cell cycle. However, mechanistically, this association remains to be elucidated. The data of the present study provide evidence that both proteins act within the same network to drive proliferation of adipose tissue stem and precursor cells, senescence, and increased risk of T2D, respectively.

Acknowledgments We thank the Department of General and Vascular Surgery, Clinical Center Bremen-Nord, for providing human abdominal subcutaneous adipose tissue. We also thank Frauke Meyer and Alisa Ivanov for technical assistance.

Conflict of interest Dominique Nadine Markowski, Helge Wilhelm Thies, Andrea Gottlieb, Heiner Wenk, Manfred Wischnewsky, and Jörn Bullerdiek declare that they have no conflict of interest.

\section{References}

Ahima RS (2009) Connecting obesity, aging and diabetes. Nat Med 15:996-997

Ashar HR, Fejzo MS, Tkachenko A et al (1995) Disruption of the architectural factor HMGI-C: DNA-binding AT hook motifs fused in lipomas to distinct transcriptional regulatory domains. Cell 82:57-65
Ayoubi TA, Jansen E, Meulemans SM et al (1999) Regulation of HMGIC expression: an architectural transcription factor involved in growth control and development. Oncogene 18:5076-5087

Bartuma H, Panagopoulos I, Collin A et al (2009) Expression levels of HMGA2 in adipocytic tumors correlate with morphologic and cytogenetic subgroups. Mol Cancer 8:36

Battista S, Fidanza V, Fedele M et al (1999) The expression of a truncated HMGI-C gene induces gigantism associated with lipomatosis. Cancer Res 59:4793-4797

Dayeh TA, Olsson AH, Volkov P et al (2013) Identification of CpGSNPs associated with type 2 diabetes and differential DNA methylation in human pancreatic islets. Diabetologia 56:1036-1046

Hennig Y, Wanschura S, Deichert U et al (1996) Rearrangements of the high mobility group protein family genes and the molecular genetic origin of uterine leiomyomas and endometrial polyps. Mol Hum Reprod 2:277-283

Kazmierczak B, Meyer-Bolte K, Tran KH et al (1999) A high frequency of tumors with rearrangements of genes of the HMGI(Y) family in a series of 191 pulmonary chondroid hamartomas. Genes Chromosomes Cancer 26:125-133

Kohonen T (2001) Self-Organizing Maps. Third, extended edition. Springer Berlin, Heidelberg, New York

Markowski DN, von Ahsen I, Nezhad MH et al (2010) HMGA2 and the p19Arf-TP53-CDKN1A axis: a delicate balance in the growth of uterine leiomyomas. Genes Chromosomes Cancer 49:661-668

Markowski DN, Winter N, Meyer F et al (2011a) p14Arf acts as an antagonist of HMGA2 in senescence of mesenchymal stem cellsimplications for benign tumorigenesis. Genes Chromosomes Cancer 50:489-498

Markowski DN, Helmke BM, Meyer F et al (2011b) BMP4 increases expression of HMGA2 in mesenchymal stem cells. Cytokine $56: 811-816$

Minamino T, Orimo M, Shimizu I et al (2009) A crucial role for adipose tissue p53 in the regulation of insulin resistance. Nat Med 15:1082-1087

Ohshige T, Iwata M, Omori S et al (2011) Association of new loci identified in European genome-wide association studies with susceptibility to type 2 diabetes in the Japanese. PLoS ONE 6:e26911

Pasquali D, Pierantoni GM, Fusco A et al (2004) Fenofibrate increases the expression of high mobility group AT-hook 2 (HMGA2) gene and induces adipocyte differentiation of orbital fibroblasts from Graves' ophthalmopathy. J Mol Endocrinol 33:133-143

Rathmann W, Haastert B, Icks A et al (2003) High prevalence of undiagnosed diabetes efficient screening. The KORA survey 2000. Diabetologia 46:182-189

Rogalla P, Lemke I, Kazmierczak B et al (2000) An identical HMGICLPP fusion transcript is consistently expressed in pulmonary chondroid hamartomas with $\mathrm{t}(3 ; 12)(\mathrm{q} 27-28 ; \mathrm{q} 14-15)$. Genes Chromosomes Cancer 29:363-366

Schoenmakers EF, Wanschura S, Mols R et al (1995a) Recurrent rearrangements in the high mobility group protein gene, HMGI$\mathrm{C}$, in benign mesenchymal tumors. Nat Genet 10:436-444

Schoenmakers EF, Geurts JM, Kools PF et al (1995b) A 6-Mb yeast artificial chromosome contig and long-range physical map encompassing the region on chromosome $12 \mathrm{q} 15$ frequently rearranged in a variety of benign solid tumors. Genomics 29:665-678

Staats B, Bonk U, Wanschura S et al (1996) A fibroadenoma with a $\mathrm{t}(4 ; 12)$ (q27;q15) affecting the HMGI-C gene, a member of the high mobility group protein gene family. Breast Cancer Res Treat 38:299-303 
Tallini G, Dal Cin P, Rhoden KJ et al (1997) Expression of HMGI-C and $\mathrm{HMGI}(\mathrm{Y})$ in ordinary lipoma and atypical lipomatous tumors: immunohistochemical reactivity correlates with karyotypic alterations. Am J Pathol 151:37-43

Tchkonia T, Morbeck DE, Von Zglinicki T et al (2010) Fat tissue, aging, and cellular senescence. Aging Cell 9:667-684

Thies HW, Nolte I, Wenk H et al (2013) Permanent activation of HMGA2 in lipomas mimics its temporal physiological activation linked to the gain of adipose tissue. Obesity. doi:10.1002/oby. 20137
Voight BF, Scott LJ, Steinthorsdottir V et al (2010) Twelve type 2 diabetes susceptibility loci identified through large-scale association analysis. Nat Genet 42:579-589

Zeggini E, Weedon MN, Lindgren CM et al (2007) Replication of genome-wide association signals in UK samples reveals risk loci for type 2 diabetes. Science 316:1336-1341

Zhou X, Benson KF, Ashar HR et al (1995) Mutation responsible for the mouse pygmy phenotype in the developmentally regulated factor HMGI-C. Nature 376:771-774 